\title{
Interfaces
}

INTERFACES Image Texte Language

\section{Ouverture synesthésique sur la poésie mystique persane : étude des sens dans la poésie de Rûmi}

\section{Leila Ghalehtaki}

\section{(2) OpenEdition}

1 Journals

\section{Édition électronique}

URL : http://journals.openedition.org/interfaces/242

DOI : 10.4000/interfaces. 242

ISSN : 2647-6754

Éditeur :

Université de Bourgogne, Université de Paris, College of the Holy Cross

\section{Édition imprimée}

Date de publication : 1 janvier 2015

Pagination : 251-260

ISBN : 9780983175247

ISSN : 1164-6225

\section{Référence électronique}

Leila Ghalehtaki, «Ouverture synesthésique sur la poésie mystique persane : étude des sens dans la poésie de Rûmi », Interfaces [En ligne], 36 | 2015, mis en ligne le 01 janvier 2018, consulté le 07 janvier 2021. URL : http://journals.openedition.org/interfaces/242 ; DOI : https://doi.org/10.4000/interfaces. 242

Les contenus de la revue Interfaces sont mis à disposition selon les termes de la Licence Creative Commons Attribution 4.0 International. 


\title{
OUVERTURE SYNESTHÉSIQUE SUR LA POÉSIE MYSTIQUE PERSANE ÉTUDE DES SENS DANS LA POÉSIE DE RÛMI
}

\author{
Leila Ghalehtaki
}

Grand poète mystique persan du XIIIe siècle, Djalal-ûd-Dîn Mohammad Rûmi (connu sous le nom de Mawlânâ - Notre Maître - en Iran), est le créateur d'une œuvre immense, d'une poésie riche de symboles et de thèmes littéraires modernes. Avant de devenir un mystique amoureux, il était un jurisconsulte et professeur dont les cours étaient fréquentés par de nombreux étudiants. En effet, ce qui provoque cette véritable transformation, c'est sa rencontre avec une personne étrange, Shams Tabrîzî, en 1244. Derviche de Tabrîz, plus tard maître de Rûmi, Shams (mot arabe signifiant le soleil) lui représente le Bien-Aimé divin et apparaît constamment dans l'œuvre du poète. Le Divan-e Shams de Tabrîz porte le nom du maître. L'insistance du poète mystique sur l'importance de la première rencontre dans la naissance spirituelle de l'homme par l'Amour divin vient en effet de cette rencontre merveilleuse et bouleversante :

J'étais le Zâhid (ascète) de mon pays, j'avais des minbars (chaires magistrales), Mais le destin du cœur m'a changé en amoureux et applaudissant de toi. ${ }^{1}$

(Rûmi, Divân-e Shams, ghazal' 2152)

Il précise par ailleurs que Shams a revivifié son âme déjà morte et l'a rendue éternelle:

J'étais mort, je suis vivant, j'étais pleurant, je suis riant,

La fortune de l'amour est venue, et je suis devenu la fortune éternelle.

(Rûmi, Divân-e Shams, ghazal 1393)

1 La traduction du persan des poèmes du Divân-e Shams de Rûmi, cités dans cet article, a été faite par la grande iranologue française et spécialiste de la poésie de Rûmi, Éva de Vitray-Meyerovitch, dans ses différents livres et essais sur la poésie mystique et Rûmi, mentionnés dans la bibliographie.

2 En tant que l'une des thématiques les plus importantes de la poésie persane, l'amour devait sa présence à la poésie lyrique dans laquelle il était célébré sous la forme de « ghazal ». « Le ghazal est un poème fait de distiques (beyt) qui tendent, chacun, à être un poème. Poème dans un poème, ils concourent à créer un monde propre à ce dernier. On en image les possibilités. La construction est très solide», cité par Charles-Henri de Fouchécour, Le Divân de Hâfez de Chiraz, Introduction, traduction du persan et commentaire, Paris, Éditons Verdier, 2006, p. 10. 
Rûmi parvient à exprimer cette transformation spirituelle ainsi que ses autres expériences spirituelles à travers une riche poésie. On est frappé par les descriptions minutieuses des états d'âme d'un mystique sous l'extase et même par les termes qu'il utilise pour les décrire. Sa poésie est pleine de comparaisons et de métaphores synesthésiques comme on va le voir. Mais, elle est également constellée des contes et récits et parfois même de récits dans les récits. Rûmi a poussé la poésie mystique persane à son apogée et a montré le chemin aux poètes persans des siècles suivants.

Avant de parler de la poésie de Rûmi et des effets synesthésiques qui la constituent en tant que telle, il faut noter que la poésie mystique persane est, comme on le dit souvent, une réaction face à la destruction totale et à la ruine de l'Iran liées aux invasions mongoles. Cette poésie vise à trouver un refuge dans le monde spirituel en accordant la priorité à l'émerveillement et à la vision au détriment de la raison et de la rationalité. Ce qui permet, comme le croient les poètes mystiques, de découvrir le «moi caché» afin de parvenir à la valeur originelle de l'homme en tant que Calife de Dieu sur la terre (dont le symbole est « l'Homme parfait» ou «Insân-ol-Kâmil»). La naissance de l'Homme parfait résulte en effet du mariage mystique de l'âme avec l'objet de son Amour. (De Vitray-Meyerovitch, Mystique et Poésie en Islam : Djalâl-ud-Dîn Rûmî et l'Ordre des Derviches Tourneurs, 241). Autrement dit, « le but ultime de l'expérience spirituelle peut être représenté comme une transmutation de l'homme « terrestre » en homme « céleste ». ${ }^{3}$ Cette transmutation entraîne inéluctablement une transformation des moyens humains de la connaissance, c'est-à-dire les cinq sens corporels. En fait, en quittant l'univers sensible et humain afin d'entrer dans le Trône du Bien-Aimé divin et de s'unir à Lui, le mystique ne pourrait plus se confier aux sens physiques. Il profite des cinq sens spirituels à travers la transmutation des sens. Rûmi en parle dans toute son œuvre immense à travers des comparaisons, métaphores et symboles comme dans l'extrait suivant:

Le sens physique est l'échelle pour ce monde ; le sens spirituel est l'échelle pour le ciel.

Recherche le bien-être du sens physique auprès du médecin ; implore le bien-être du sens spirituel auprès du Bien-Aimé.

La santé de celui-là provient de l'état florissant du corps ; la santé de celui-ci provient de la ruine du corps.

(Rûmi, Mathnawî, Livre premier, vers 303-3054)

3 Ibid.

4 La traduction du persan de Mathnawî a été faite par Éva DE VITRAY-MEYEROVITCH et Djamchid MORTAZAVI, La Quête de l'Absolu, Paris : Éditions du Rocher, 1990 
L'assimilation des sens corporels aux « escaliers terrestres » et des sens spirituels aux « escaliers célestes » montre la différence fondamentale des deux sens et leur importance dans l'élévation de l'homme dans les deux différents univers.

L'idée rûmienne de la « transmutation des sens » nous fait penser à l'idéal rimbaldien d'être « voyant » qui se réalise à travers « un long, immense et raisonné dérèglement de tous les sens » (Rimbaud 251). Ainsi, sans nous préoccuper des différentes philosophies et visions de ces deux grands poètes, l'un mystique, l'autre humaniste, il semble que chez Rimbaud, le poète doit revêtir les caractéristiques de «l'Homme parfait » ou "insân-ol-kâmil », l'homme idéal de Rûmi. Comme l'homme rûmien qui désire s'unir à l'Âme Unique dont il fait partie pour ne parler que Son langage, ne voir qu'Elle et n'entendre que Sa voix, l'homme rimbaldien voudrait être « voyant », " se faire voyant » pour découvrir cet « autre » de lui-même, pour « le regarder » et pour « l'écouter ». L'homme rimbaldien ( « le cuivre ») est à la recherche de son âme ( "l'inconnu » ou « le clairon »), tout comme l'homme rûmien désire changer le « cuivre » de son existence en « or » (symbole de l'Âme Unique) grâce à l'alchimie de l'Amour. Cette transformation se fait à travers une transmutation des sens. Dans le grand Mathnawî, Rûmi dit :

Attends patiemment que tes sens corporels soient transmués, afin que tu puisses voir ce qui est caché et que la difficulté soit résolue.

(Rûmi, Mathnawî, Livre premier, vers 1039)

Il est à signaler que pour le Maître mystique, l’homme possède en plus de ses sens corporels, comparés au « cuivre ", cinq sens spirituels ${ }^{5}$, comparés à « l'or rouge » (Mathnawî̀, Livre deuxième, vers 49). Selon Rûmi, les sens corporels s'enracinent dans l'obscurité, tandis que les sens spirituels s'enracinent dans le soleil. ${ }^{6}$ L'assimilation des sens corporels au cuivre enraciné dans l'obscurité et des sens spirituels à l'or enraciné dans le soleil s'accentue aussi métaphoriquement par la différence entre l'objet et son ombre. Ainsi, affirme-t-il : « Sache que l'œil externe n'est que l'ombre de cet œil intérieur [...] (Livre deuxième, vers 611). Puisque notre monde matériel n'est que l'ombre d'un vrai monde caché, ce qui nous rappelle l'idée platonicienne, les sens corporels aussi sont l'ombre des sens spirituels.

5 Le poète emploie aussi l'expression de «cinq sens externes » et " cinq sens internes ». Cf. Mathnawî, Livre premier, 3574 .

$6 \quad$ Ibid., vers 47,49 et 51 
Mais il faut être attentif au fait que Rûmi insiste sur la transmutation des sens corporels et non pas sur leur effacement. Rûmi adopte différentes positions envers les deux sens selon l'importance du rôle qu'ils jouent dans l'accomplissement de la démarche mystique. Or, tantôt Rûmi conseille d'ignorer les sens corporels en les taxant d'être incompatibles avec la démarche mystique et l'Union au BienAimé divin, tantôt, sans rejeter ces derniers, il insiste sur la coexistence et la collaboration des deux sens. Mais, au bout du compte, il donne la priorité aux sens spirituels. Il affirme : " Laisse vaguer ton esprit, et puis sois attentif. Bouche tes oreilles, et puis écoute » (Livre troisième, vers 1291).

Mais comment parvient-on à cette transformation ? Rûmi croit que la transmutation des sens s'effectue à travers l'alchimie qu'exerce Dieu à travers la grâce divine. C'est par Dieu que les sens changent. Rûmi l'affirme ainsi :

Partout où il y avait une oreille, par Lui, elle devient un œil [...].

(Rûmi, Mathnawî, Livre premier, vers 515)

Il est bien connu que le cuivre par l'alchimie devient de l'or. Notre cuivre a été transmué par cette merveilleuse alchimie.

(Rûmi, Divân-e Shams, tarji'ât 23)

Cependant, le Maître mystique croit à l'homme et à sa volonté d'atteindre ou d'accéder à cette transmutation.

Sois à la recherche de la transmutation.

Contemple les alchimistes du Ciel ; écoute à chaque instant les paroles qui proviennent des fabricants spirituels de la pierre philosophale.

(Rûmi, Mathnawî, Livre quatrième, vers 3074)

Ce qui distingue Rûmi et ses successeurs, c'est qu'ils ont une forte croyance au pouvoir de l'homme et en sa capacité d'atteindre au plus haut degré de perfection spirituelle. En effet, cette idée ne se trouve pas seulement chez les mystiques persans, mais on la voit aussi dans l'œuvre d'un grand sage et réformiste du XIIIe siècle comme Saadi quand il dit :

L'homme peut atteindre au degré où il ne voit plus rien que Dieu :

Considère à quelle grandeur parvient l'état d'humanité ! 
Mystique ou sage, ces grands poètes persans sont connus pour leur pur humanisme et leur insistance sur la place de l'homme et son pouvoir de tout changer dans le monde.

En ce qui concerne les sens, il est à noter que chez Rûmi, nous trouvons des vers marquant l'influence des sens l'un sur l'autre. Ainsi, par exemple, celle du sens olfactif sur le sens visuel. Cela apparaît surtout dans les thèmes liés à l'histoire de la Séparation de Jacob d'avec son fils, Joseph. Je cite Rûmi : « L'odeur nauséabonde obscurcit l'œil, l'odeur de Joseph aide les yeux » (Livre Premier, vers 1903).

De plus, certains mystiques comme Rûmi croient à la substitution des sens, ce qui veut dire qu'un sens pourrait jouer le rôle d'un autre sens. Alors, le sens auditif pourrait céder le pas au sens olfactif. Ce qu'on peut voir partout dans la poésie persane et par exemple la poésie de Hâfez de Chirâz lorsqu'il dit :

Qui a entendu le souffle du zéphyr Ton agréable parfum,

A entendu de son Compagnon intime le souffle familier.

(Hâfez, Divân, ghazal 2387)

En effet, aujourd'hui même, dans le persan populaire, on emploie les verbes de perception sensorielle l'un pour l'autre. Par exemple, on remplace les verbes indiquant les deux sens olfactif et visuel. On dit par exemple : «Tu entends cette odeur? », ou « Tu vois comme ça sent bon ? ». De même, en attirant l'attention de l'autre sur ce qu'on va dire, on emploie le verbe « regarder» au lieu du verbe « écouter».

Maître mystique, Rûmi insiste sur le remplacement des sens dans la démarche mystique. Nous voyons surtout le remplacement du sens auditif par le sens visuel. Je cite Rûmi :

Chaque fois qu'une réponse parvient au cœur à travers l'oreille, l'œil dit : « Entends cette réponse de moi ; ne fais pas attention à celle-là (qui provient de l'oreille). » (Livre deuxième, vers 857)

Quand l'oreille est pénétrante, elle devient l'œil : sinon, la parole de Dieu reste emmêlée dans l'oreille sans atteindre le cœur. (Livre deuxième, vers 862)

Le poète mystique exprime la cause de son insistance en affirmant :

L'oreille est une entremetteuse, tandis que l'œil connait l'union ; l'œil possède une expérience directe (de la réalité), tandis que l'oreille n'a que des mots.

Traduction du persan par Charles-Henri DE FOUCHÉCOUR, Paris, Éditons Verdier, 2006. 
Lorsque l'oreille entend, il en découle une transformation des qualités ; dans la vision de l'œil, il y a une transformation de l'essence. (Livre deuxième, vers 858, 859)

Donc, la transformation du sens auditif en sens visuel vise à changer l'hésitation en observation et certitude comme le précise un proverbe persan dont l'équivalent français est: « Il faut le voir pour le croire ».

Aussi, trouvons-nous des vers qui indiquent la transformation du sens auditif en sens gustatif: « C'est un 'Me voici' que tu ne peux pas entendre, mais que tu peux savourer de la tête jusqu'aux pieds » (Livre deuxième, vers 1191). Savourer l'appel de Dieu pour le mystique au lieu de l'entendre, c'est accepter l'appel du Bien-Aimé avec toute l'existence fragile et mortelle de celui-ci.

Au-delà de ces remplacements ou ces transformations des sens les uns dans les autres, Rûmi aborde dans son œuvre le rôle des sens dans la démarche mystique. Jetons-y un rapide coup d'œil :

1. Le sens auditif entre en jeu quand le mystique « entend» la voix de l'Aimé et la musique divine déjà entendue dans son pays originel, le paradis, avant sa chute sur la terre. Ce qui rappelle l'évocation de l' « anamnèse » platonicienne. La « naissance spirituelle », but de la mystique, se réalise au moment où l'amant mystique « entend » l'appel du Bien-Aimé. Rûmi en parle ainsi :

Je mourus cent fois et je trébuchai,

Mais je renaquis dès que j'entendis Ta voix.

(Rûmi, Divân-e Shams, ghazal 1689)

Nous étions morts et complètement détruits ; l'appel de Dieu est venu, nous nous sommes tous levés.

(Rûmi, Mathnawî, Livre premier, vers 1933)

Une voix retentit dans le néant. Le néant dit: « Oui !» ${ }^{8}$

Je mets le pas au-delà, tout jeune, vert et joyeux.

(Rûmi, Divân-e Shams, ghazal 1832)

Ces vers abordent la notion de temps mystique. Temps du « néant», le temps passager se termine par l'appel du Bien-Aimé. L'amant entend l'appel de Celui-ci, y répond par un « oui » et reprend vie. Par

8 Allusion au « Mithâq coranique » ou la «Pacte de la prééternité » (Alast). 
ce « oui », le temps mystique prend son essor et commence son mouvement ascendant vers le Trône céleste du Bien-Aimé, vers l'éternité.

2. Le sens olfactif. La « naissance spirituelle » de l'amant mystique se réalise aussi à travers la perception du parfum ou de l'odeur du Bien-Aimé divin. Il suffit d'entendre c'est-à-dire de sentir le parfum divin pour que l'amant naisse et qu'il soit ivre de l'Amour. Je cite Rûmi :

Le jour où l'âme prend son essor, enivré par ton parfum,

L'âme connaîtra, l'âme connaîtra le parfum du Bien-Aimé.

(Rûmi, Divân-e Shams, ghazal 622)

Du Trône de l'Invisible proviennent une nuée, un parfum :

Ô vous, les initiés secrets, prenez votre essor vers ce parfum!

(Rûmi, Mathnawî, Livre premier, vers 825)

3. Le sens visuel apparaît sous son aspect amoureux c'est-à-dire à travers le regard amoureux. Étant inaugural et doué d'un pouvoir générant, le regard amoureux fait renaître spirituellement l'amant mystique dans le moment béni de «waqt» («l'instant présent») dans lequel se passe l'Union mystique. Nous lisons chez Rûmi : «Lorsque je Le vis pour la première fois, Il me donna la vie [...] » (Divân-e Shams, ghazal 358).

4. Le sens gustatif apparaît à travers le thème de l'ivresse mystique. Dans la vision mystique, l'amour est symbolisé par le vin divin ou par l'Eau de la Vie par allusion à la source d'eau vive qui rend immortel celui qui en boit. L'ivresse et la vision mystique conduisent toutes les deux à la possession de Dieu. Le vin divin ou l'Eau de la Vie, tout en irriguant le mystique assoiffé, le rendent éternel. A Dieu, le poète dit :

Tu es l'eau, nous sommes pareils au champ assoiffé,

Ne détourne pas Ton visage de nous, car Tu es l'eau délectable.

(Rûmi, Divân-e Shams, ghazal 2692)

Et à son disciple, il dit :

Dieu te donne une gorgée de vin, tel que celui qui est enivré par Lui échappe aux deux mondes.

(Rûmi, Mathnawî, Livre quatrième, vers 2683) 
5. Le sens tactile, le dernier sens, c'est un sens qui a plus de rapports avec les perceptions physiques donc incompatible avec l'univers immatériel de la mystique. Cependant, il semble qu'il apparaît à travers la brûlure par le feu de l'Amour divin, celle qui entraîne l'absorption et la fusion de l'âme humaine avec l'Âme Unique et finit par lui donner l'éternité. Donc, le sens le plus important. Rûmi exprime ainsi l'importance de ce sens :

Si ta connaissance du feu n'a été transformée en certitude que par des mots, cherche à être cuit (par le feu même) et ne demeure pas dans la certitude d'une connaissance venant d'autrui.

Il n'existe point de certitude intuitive avant de brûler ; si tu désires cette certitude, mets-toi dans le feu.

(Livre deuxième, vers 860-861)

Le mystique se compare donc à une salamandre, " Oiseau mythique qui mange du feu et qui est le symbole de l'amour ardent et brûlant ». L'amant est une salamandre qui se brûle dans le feu de l'Amour afin d'atteindre à l'union amoureuse. Chez Rûmi ce symbole fait allusion à « l'Homme parfait ». Rûmi invite ses disciples à se jeter dans ce feu heureux :

Salut, ô vous dont les âmes sont semblables aux phalènes, orientez-vous sur ce feu

Puisqu'au commencement vous avez dit « Oui », acceptez le malheur.

Il est semblable à la salamandre qui demeure dans le feu

Celui qui a dans le cœur et dans l'âme un tel désir et un tel amour.

(Divân-e Shams, ghazal 142)

Cette brûlure offre l'éternité à celui qui l'accepte comme le prophète Abraham :

Viens ici, et vois les mystères d'Abraham, qui dans le feu trouva des cyprès et des jasmins.

(Rûmi, Mathnawî, Livre premier, vers 790)

Dans la fournaise de l'amour, tu es comme Abraham :

$\mathrm{Ne}$ crains rien, il ne te laissera pas périr. 
A la fin et grâce à cette brûlure, se réalise l'Union de l'amant avec le Bien-Aimé pour que n'existe plus un Bien-Aimé et un amant séparés, mais une seule unité. Uni à l'Aimé, absorbé dans « l'Unique Essence », l'amant ne s'appuie plus sur ses sens corporels et ne dit plus ses propres mots : sa parole est celle de Dieu. De là vient la fameuse assimilation rûmienne de l'Homme parfait à une flûte de roseau (l'instrument traditionnel iranien de musique), jouée par celui-ci mais qui émet et transmet les paroles de Dieu. C'est le cas d'al-Ḥallâj. Rûmi le considère comme " l'Homme parfait » (Mathnawî, Livre cinquième, vers 2038) dont la parole est celle de Dieu. Ainsi, le «Je suis Dieu » ("Ana al-Haqq») d'al-Hallâj signifie «Il est Dieu » («Howal-Haqq»). (Zohreh Kassaîan et les autres 263). L'homme uni au Bien-Aimé divin ne voit et n'entend que Celui-ci, et ne parle que les mots de Ce dernier. La fusion de l'Âme de l'Homme parfait dans l'Âme Unique crée une nouvelle existence devant laquelle un grand mystique persan du Xe siècle, Abû Saîd Abi'l-khayr, dont l'influence sur les mystiques persans est incontestable, s'étonne en disant :

Mon corps se transforma en larmes, mon œil versa des larmes

Il faut vivre dans ton amour sans corps.

Il ne reste rien de moi, qu'est-ce que cet amour?

Maintenant que je suis le Bien-Aimé, qui est donc l'amant?

(Abû Saîd Abil-khayr, Les Distiques, vers 131)

En conclusion, je souligne brièvement que la fusion des sens ou la synesthésie, idée si chère aux poètes et écrivains du monde, trouve, semble-t-il, ses racines, tout d'abord, en Occident, chez Platon, Plotin et les néo-platoniciens, à travers l'idée de la «transmutation des sens », et plus tard, en Orient, comme nous venons de voir, chez les poètes de la Perse et surtout ses poètes mystiques, à travers l'idée rûmienne des " cinq sens spirituels ", pour apparaître de nouveau chez les poètes occidentaux parmi lesquels Rimbaud à travers son « dérèglement des tous les sens ». Cela nous révèle en effet un échange constant entre l'Orient et l'Occident en ce qui concerne la synesthésie ou la fusion des sens. Autrement dit, ni l'époque, ni la distance géographique, ni les différences de la religion, ne changent l'imaginaire humain ou les rêveries de l'homme envers les sens ou leur fusion. Cette idée affirme en effet l'idéal goethéen de «Weltliterature » ou littérature universelle, une totalité heureuse de la pensée et de la littérature provenant du même imaginaire humain. C'est ainsi que, malgré l'absence probable d'un contact direct, les images liées à la synesthésie pourraient exister dans presque tous les textes littéraires du monde et les poèmes de Rûmi semblent en être un des meilleurs exemples du Moyen Age. 


\section{Ouvrages Cités}

BARAHANI, Reza. « Mawlavi, Surréalisme, Rimbaud et Freud », in Revue de la Faculté des Lettres de Tabrîz, Année 17, n² 2, Été 1965, pp. 221-240

HÂFEZ DE CHIRÂZ. Le Divân, CEuvre lyrique d'un spiritual en Perse au XIVème siècle, Introduction, traduction du persan et commentaire, par Charles-Henri DE FOUCHÉCOUR, Paris : Éditons Verdier, 2006.

PAGEAUX, Daniel-Henri. La littérature générale et comparée, Paris : Éditions Armand Colin, Collection Cursus, série « Littérature », 1994.

POUILLON, François. «Arthur Rimbaud », in Dictionnaire des orientalistes de langue française, Paris : Éditions KARTHALA, 2008.RIMBAUD, Arthur. «Lettre du voyant », in CEuvres complètes, Paris : Gallimard, pp. 250-251

RÛMI, Djalâl-UD-Dîn Mohammad (MAWLÂNÂ). Divân-e Shams-e Tabrîzî, 2. Vol., Téhéran : Éditions Negâh, 2008.

RÛMI, Djalâl-UD-Dîn Mohammad (MAWLÂNÂ). Mathnawî, Téhéran : Éditions Zavvareh, 1990.

RÛMI, Djalâl-UD-Dîn Mohammad (MAWLÂNÂ). Mathnawî, la Quête de l'Absolu, Traduction du persan par Éva DE VITRAY-MEYEROVITCH et Djamchid MORTAZAVI, Paris : Éditions du Rocher, 1990.

SADJDJÂDI, Dja'far. Farhang-e Estelahat va Ta'birât-e Erfani (Glossaire des termes et expressions mystiques), Téhéran : Éditions Tahouri, 2010.

TAJODDINI, Ali. Farhang-e nemâd-hâ va neshâneh-hâ dar andisheye Mawlânâ (Dictionnaire des symboles et des signes dans la pensée de Mawlânâ), Téhéran : Éditions Soroosh, 2009.

VITRAY-MEYEROVITCH, Éva de. Rûmi : Le Chant du Soleil, Paris : Éditions La Table Ronde, Coll. Les petits livres de la sagesse, 1997.

VITRAY-MEYEROVITCH, Éva de. Mystique et Poésie en Islam : Djalâl-ud-Dîn Rûmî et l'Ordre des Derviches tourneurs, Paris : Editions Desclée de Brouwer, 1972.

ZAMANI, Karim. Shrh-e Jâme '-e Mathnawî-e Ma'nawî, Daftar-e avval (Analyse intégral de Mathnawî, Livre premier), Téhéran : Éditions Ettelâ'ât, 2010. 